\title{
Novel Approach of Fragment-Based Lead Discovery applied to Renin Inhibitors
}

Michiko Tawada, ${ }^{* \dagger}$ Shinkichi Suzuki, ${ }^{\dagger}$ Yasuhiro Imaeda, ${ }^{\dagger}$ Hideyuki Oki, ${ }^{\dagger}$ Gyorgy

Snell,,$^{\ddagger}$ Craig A. Behnke,,$^{+S}$ Mitsuyo Kondo, ${ }^{\dagger}$ Naoki Tarui,$^{\dagger}$ Toshimasa Tanaka, ${ }^{\dagger}$

Takanobu Kuroita, ${ }^{\dagger}$ and Masaki Tomimoto ${ }^{\dagger}$

$†$ Pharmaceutical Research Division, Takeda Pharmaceutical Company, Ltd.,

2-26-1, Muraoka-higashi, Fujisawa, Kanagawa 251-8555, Japan

\$Takeda California, Inc., 10410, Science Center Drive, San Diego, California

92121, United States

\section{*Corresponding author}

Phone: +81-466-32-2704

Fax: +81-466-29-4434

E-mail: michiko.tawada@takeda.com 


\title{
Present address
}

$\S 9363$ Towne Centre Drive, San Diego, California 92121, United States

\section{Key words}

Fragment-based drug discovery (FBDD), high-throughput synthetic chemistry, structure-based drug design (SBDD), renin inhibitor, crystal structure

\begin{abstract}
A novel approach was conducted for fragment-based lead discovery and applied to renin inhibitors. The biochemical screening of a fragment library against renin provided the hit fragment which showed a characteristic interaction pattern with the target protein. The hit fragment bound only to the $\mathrm{S} 1, \mathrm{~S} 3$, and $\mathrm{S} 3{ }^{\mathrm{SP}}$ (S3 subpocket) sites without any interactions with the catalytic aspartate residues (Asp32 and Asp215 (pepsin numbering)). Prior to making chemical modifications to the hit fragment, we first identified its essential binding sites by utilizing the hit fragment's substructures. Second, we created a new and smaller scaffold, which better occupied the identified essential $\mathrm{S} 3$ and $\mathrm{S} 3{ }^{\mathrm{SP}}$ sites, by utilizing library synthesis with high-throughput chemistry. We then revisited the S1 site and efficiently explored a good building block attaching to the scaffold with library
\end{abstract}


synthesis. In the library syntheses, the binding modes of each pivotal compound were determined and confirmed by X-ray crystallography and the library was strategically designed by structure-based computational approach not only to obtain a more active compound but also to obtain informative Structure Activity Relationship (SAR). As a result, we obtained a lead compound offering synthetic accessibility as well as the improved in vitro ADMET profiles. The fragments and compounds possessing a characteristic interaction pattern provided new structural insights into renin's active site and the potential to create a new generation of renin inhibitors. In addition, we demonstrated our FBDD strategy integrating highly sensitive biochemical assay, X-ray crystallography, and high-throughput synthesis and in silico library design aimed at fragment morphing at the initial stage was effective to elucidate a pocket profile and a promising lead compound.

\section{Graphical abstract}

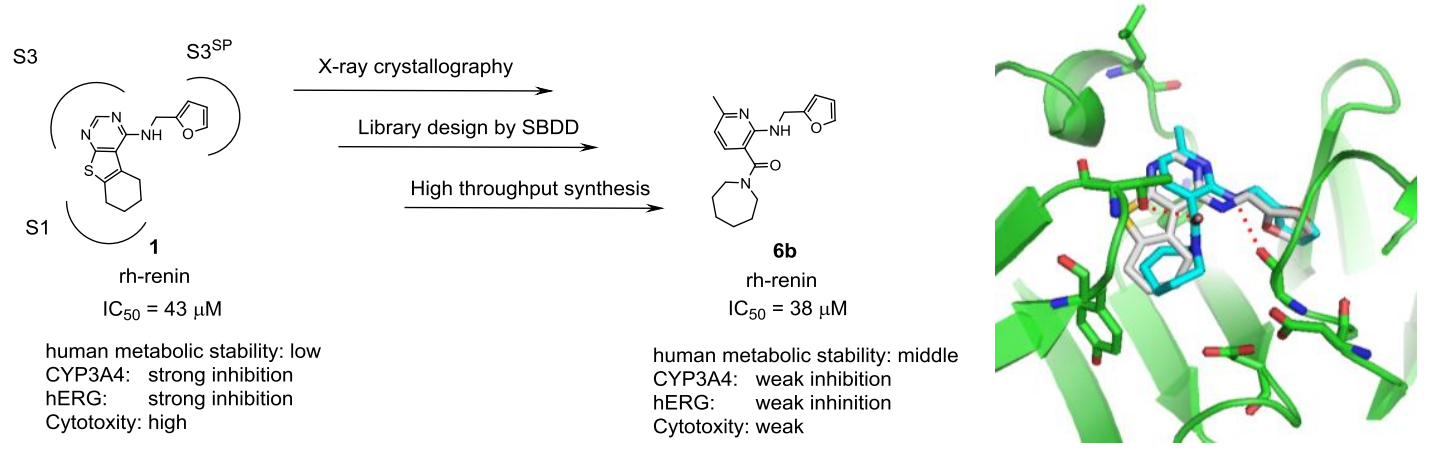




\section{Introduction}

Fragment-based drug discovery (FBDD) has emerged as an effective

methodology to generate high quality leads. The advantage of FBDD is that low-molecular-weight chemical fragments can be discovered, which bind to the target in relatively ideal contact with the active site $^{1}$. Generally, the first step of FBDD is to screen fragment compounds fitted to the site that might be essential for ligand-binding. Compared with high-throughput screening hits, the identified small molecules are expected to be optimized efficiently into a drug candidate which maintains low molecular weight and possesses both a great binding affinity and a favorable pharmacokinetic profile.

The renin-angiotensin system (RAS) is one of the major regulating systems of the arterial blood pressure in humans ${ }^{2,3}$. Renin is a member of the aspartic protease family and catalyzes the conversion of the decapeptide angiotensin I from angiotensinogen, the first reaction in the RAS. Although renin has been studied as an attractive drug target for decades and numerous crystal structures have been elucidated, it remains to be a very challenging target ${ }^{4}$. Most renin inhibitors, as represented by aliskiren, have peptide-like structures and/or high molecular weight, which result in poor 
oral bioavailability ${ }^{5,6}$. Although extensive drug discovery efforts have been dedicated to this target, only one renin inhibitor, aliskiren which exhibits the limited oral bioavailability in humans ${ }^{7-10}$, has been launched so far.

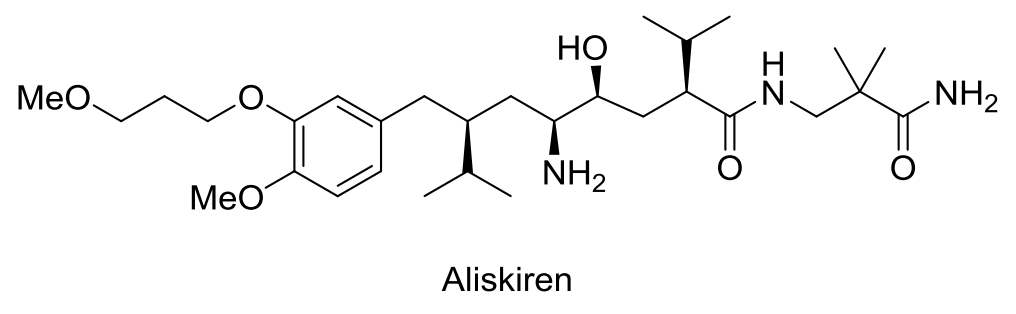

Figure 1. Structure of aliskiren.

The difficulty in medicinal chemistry is attributed to the large active site of renin.

The active site consists of distinct binding pockets which recognize the side-chains of the angiotensinogen, called the $\mathrm{S} 1, \mathrm{~S} 2, \mathrm{~S} 3$, and $\mathrm{S} 1$ ' sites, etc., and the nonsubstrate $\mathrm{S} 3{ }^{\mathrm{SP}}$ (S3 subpocket) site (Figure 2) ${ }^{11}$. Two aspartate residues, Asp32 and Asp215 (numbering based on the pepsin sequence), called the catalytic residues, locate around the center of the active site to promote the hydrolysis of amide bond of angiotensinogen. All the clinical candidates of direct renin inhibitors possess polar functional groups to interact via hydrogen-bonding with the catalytic aspartate residues such as the hydroxy group or the basic amine ring. Small molecule inhibitors which have been discovered as screening 
hits, trans-4-(4-chcorophenyl)-3-(4-methoxybenzyl)-piperidine series reported by Hoffmann-La Roche ${ }^{12}$ or 6-ethyl-2,4-diaminopyrimidine series by Pfizer $^{13}$, also form polar interactions with the catalytic aspartates. Although a long history of medicinal chemistry of renin inhibitors has shown that the $\mathrm{S} 1, \mathrm{~S} 3$, and $\mathrm{S} 3{ }^{\mathrm{SP}}$ sites act as energetically favored inhibitor binding sites, the interaction with the catalytic aspartates have been considered to be the most important and inevitable. ${ }^{4,9,14}$ From this point of view, the drug discovery process of many renin inhibitors, most of which have not been launched, might start from an identification of core scaffolds interacting with the aspartate residues. In order to generate a lower-molecular-weight renin inhibitor which is expected to have a good pharmacokinetic profile, it is likely important to identify the hot spots for a ligand-binding among the several distinct pockets and start chemical modification from a better-fitted compound in the hot spots. Using fragment-based approach, small and active site-engaging fragments are discovered and the pockets, to which such simple fragments bind, may be recognized as the hot spots. Hit discovery using FBDD technology is considered to be well suited for renin inhibitors. 

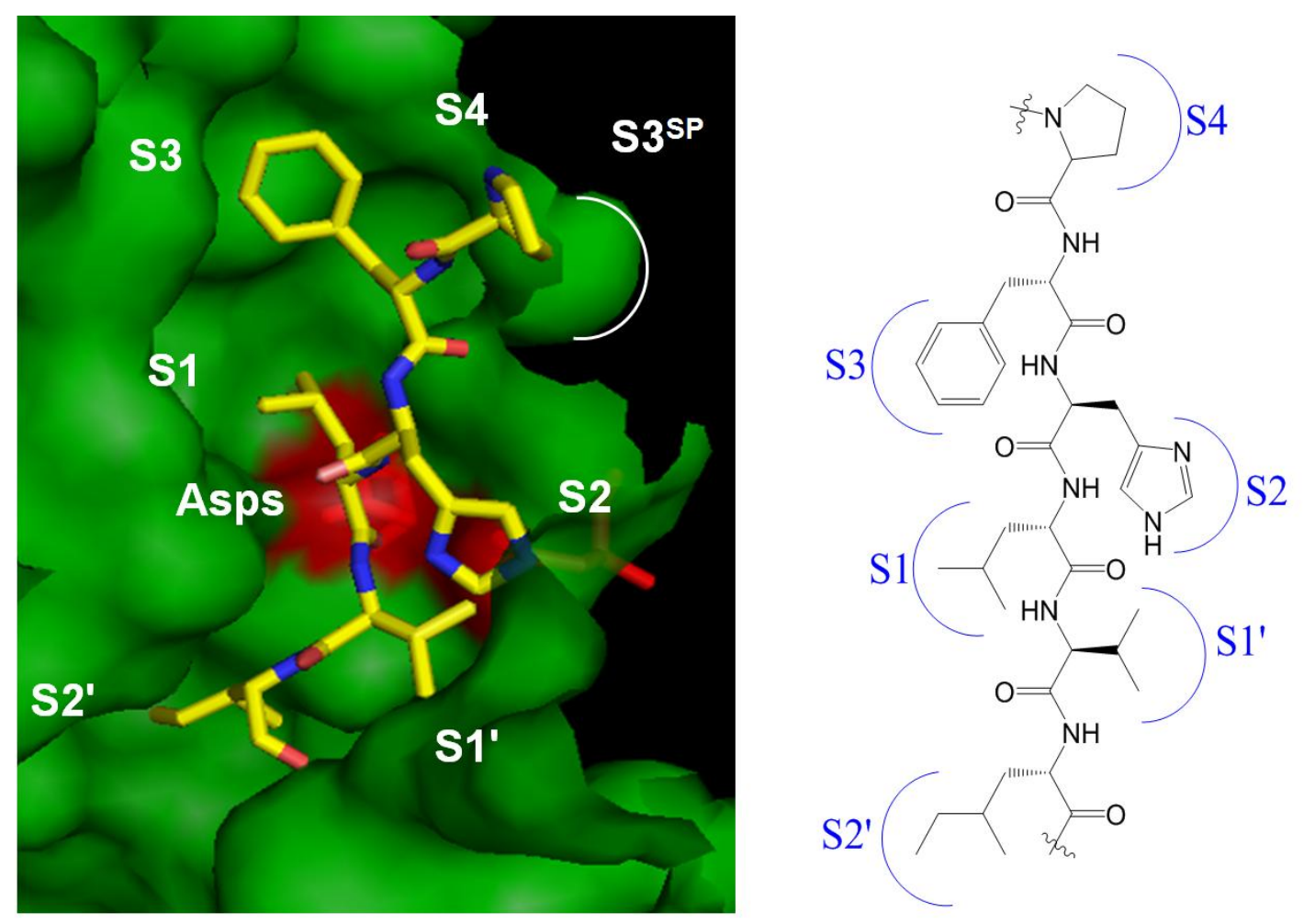

Figure 2. X-ray crystal structure of renin complexed with angiotensinogen [PDB entry 2X0B]. The protein surface is colored green and the catalytic aspartate residues (Asp32 and Asp215) are highlighted in red. On the right, the chemical structure of the 6 amino acid residues around the cleaved region of angiotensinogen is depicted together with the schematic representation of distinct binding pockets.

Hence, we applied the FBDD method to obtain a lead compound as renin inhibitors with good properties. Our hit fragment which was used as the chemical starting point possessed a characteristic interaction pattern with the target. The fragment formed efficient hydrophobic interactions with the S1 and S3 sites and the small nonsubstrate 
$\mathrm{S} 3{ }^{\mathrm{SP}}$ site, as discovered by $\mathrm{X}$-ray crystallography of the enzyme-fragment complex, without a direct interaction with the catalytic aspartate residues. As a result of FBDD in combination with structure-based computational design and high-throughput synthetic chemistry, we have successfully obtained a novel lead compound. We report here a fully detailed account of our FBDD process in the quest for a lead compound of renin inhibitors.

\section{Results and Discussions}

\section{2-1. Identification of a fragment hit and structural feature of renin binding}

The enzyme assay at a high concentration $(100 \mu \mathrm{M})$ of in-house fragment library identified 6-[(furan-2-ylmethyl)amino]pyrimidine derivative $\mathbf{1}$ as a hit fragment. The crystal structure of $\mathbf{1}$ complexed with renin showed that this compound binds to the S1 site, the $\mathrm{S} 3$ site, and $\mathrm{S} 3{ }^{\mathrm{SP}}$ site without a direct interaction with the catalytic aspartate residues (Asp32 and Asp215) (Figure 4(a)). The characteristic interactions are a hydrogen-bond between the $\mathrm{NH}$ of the furfurylamino group and the backbone carbonyl of Gly217 and the tight hydrophobic interactions with the S1 and S3 sites and the small S3 ${ }^{\mathrm{SP}}$ site. The tricyclic ring of $\mathbf{1}$ forms beneficial interactions with the surrounding hydrophobic amino acids such as Tyr75, Leu110, and Phe112, in contact with the 
contiguous $\mathrm{S} 1-\mathrm{S} 3$ site. Due to the $\mathrm{CH} / \pi$ interaction between the endocyclic saturated ring and Tyr75, the $\beta$-hairpin loop consisting of residues $72-81$, known as 'the flap ${ }^{15}$, adopts a close conformation and shields the S1 site from the solvent region. The furfurylamino group shows good shape matching with the $\mathrm{S} 3^{\mathrm{SP}}$ site which is not occupied by the natural ligand, angiotensinogen.

Interestingly, although the fragment library had been carefully designed and contained many basic fragments, none of those basic ones were identified as hit fragments. Our highly sensitive enzyme assay and technologically-advanced X-ray crystallography identified the hit fragment which possessed a characteristic interaction pattern without a direct interaction with catalytic aspartates despite the weak activity. The hit fragment demonstrated that favorable interactions with the $\mathrm{S} 1-\mathrm{S} 3$ site and the $\mathrm{S} 3{ }^{\mathrm{SP}}$ site allowed a molecule to bind to renin without engaging the catalytic aspartate residues. In other words, the interactions with the S1-S3 hydrophobic site and the small S3 ${ }^{\mathrm{SP}}$ site could provide an important driving force for ligand-binding to the active site on their own. The hit fragment 1 provided the new insight into the molecular recognition of renin. This compound was an attractive starting point for lead generation, as it has a potential to grow to a new generation of renin inhibitor which belongs to a new structural class. 


\section{2-2. $1^{\text {st }}$ library for optimization of fragments binding to the $\mathrm{S3}$ and $\mathrm{S3}{ }^{\mathrm{SP}}$ sites}

As mentioned earlier, if the essential sites for ligand-binding are identified and ideal chemical structures fitted to them were discovered, lower-molecular weight inhibitors can be generated. Compound $\mathbf{1}$ was an attractive hit compound in point of its interactions with the target protein. However, the tricyclic core was a bad vector targeting the catalytic aspartates. In addition, 1 exhibited a low solubility in JP2 (Japanese Pharmacopoeia Second fluid, $\mathrm{pH}$ 6.8) probably due to the rigid and planar conformation of the tricyclic core (Table 2). Compound 1 also showed the undesirable CYP3A4 inhibition, hERG inhibition and cytotoxicity because of its high lipophilicity $(\mathrm{Clog} \mathrm{P}=$ 3.9(Table 1,2)), which may cause the binding to various off-target proteins. Although an ordinary FBDD strategy is to start from growing and/or linking hit fragments to lead a hit/lead/drug candidate ${ }^{1}$, we first attempted to identify additional essential sites and then optimize a core structure binding to the essential site, prior to making chemical modifications of the hit fragment for targeting the catalytic residues. The chemical fragments of $\mathbf{1}$, that is the tricyclic moiety $\mathbf{2}$ and the furfurylamine $\mathbf{3}$, were chosen among commercially available compounds and assayed for the renin inhibitory activity (Figure 3). These structural components did not inhibit renin and thus we conclude that concurrent interactions with both of the $\mathrm{S} 3$ site and the $\mathrm{S} 3{ }^{\mathrm{SP}}$ site were essential for 
binding affinity. In other words, chemical components bound in both of those sites were needed for the initial hit fragment structure. Meanwhile, it was still unclear if an interaction with the S1 site is important. Although the contiguous S1-S3 sites have been considered as one of the hot spots, comparison of the importance between the S1 and S3 sites has not been elucidated clearly. Hence, we optimized the structure bound to the essential S3 site and $\mathrm{S} 3{ }^{\mathrm{SP}}$ site concurrently with confirming the importance of the $\mathrm{S} 1$ site. The NH substituted at the 6-position of the pyrimidine ring is important for a hydrogen-bond with the backbone carbonyl of Gly217. Keeping the NH, each building block bound to the $\mathrm{S} 3$ site and the $\mathrm{S} 3{ }^{\mathrm{SP}}$ site was simultaneously examined using high-throughput synthetic chemistry ${ }^{16}$. Among direct renin inhibitors, diverse structures have been found to bind to the $\mathrm{S} 1-\mathrm{S} 3$ site and the $\mathrm{S} 3{ }^{\mathrm{SP}}$ site. For example, as the $\mathrm{S} 3^{\mathrm{SP}}$ site binding element, the furfurylamino group is suitable for $\mathbf{1}$ and the methoxyethyl moiety is suitable for aliskiren. There should be the best combination of building blocks bound to the $\mathrm{S} 3$ site and the $\mathrm{S} 3{ }^{\mathrm{SP}}$ site. High-throughput synthetic chemistry is useful for the exploration of several building blocks to select the most suitable substituents at each site and obtain wide SAR data at the same time. 


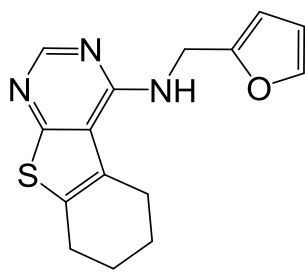

1

$\mathrm{IC}_{50}=43 \mu \mathrm{M}$

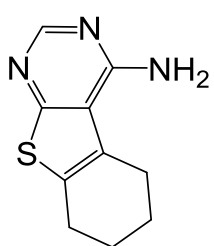

2

$\mathrm{IC}_{50}>300 \mu \mathrm{M}$

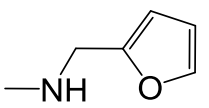

3

$\mathrm{IC}_{50}>300 \mu \mathrm{M}$

Figure 3. Fragment hit $\mathbf{1}$ and its structural components, $\mathbf{2}$, and $\mathbf{3}$

The initial library was synthesized using halide substitution by various amines

(scheme 1). The library was designed to better occupy the S1 and/or S3 site and S3 ${ }^{\mathrm{SP}}$ site using a structure-based approach in four steps: (1) a virtual library was generated by combining commercially available arylhalides and amines, (2) the virtual library was docked into renin based on the binding mode of $\mathbf{1}$, (3) their binding energies were calculated using the MM/PBSA method, ${ }^{17}$ (4) prospective structures were selected in terms of binding energies. Computational studies suggested a monocyclic core instead of the tricyclic ring was promising due to the favorable binding to the S3 site and the better vector targeting the catalytic aspartate residues. On the basis of these estimations, we enriched monocyclic cores in the initial library. In addition, diverse components were included to obtain SAR for speedy sequential chemical derivatization. 


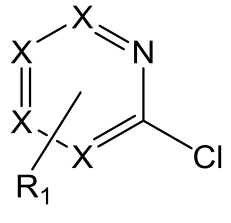

$\mathrm{X}=\mathrm{C}$ or $\mathrm{N}$<smiles>[R]CN</smiles>

$150^{\circ} \mathrm{C}$

overnight

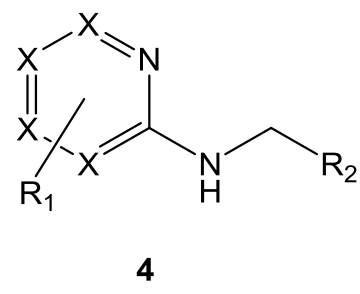

4

Scheme 1. Synthesis of the initial library using halide substitution by amines

From the initial library, the simple monocyclic core compounds which reduced the molecular size of hit fragment $\mathbf{1}$ by about $25 \%$ were found to bind to renin keeping the potency at the same level. Several monocyclic core compounds exhibited approximately tens of micromolar renin inhibitory activity, as represented by the 2-chloropyrazine derivative 4a, which exhibited the inhibitory activity of $65 \%$ at a concentration of $100 \mu \mathrm{M}$ (Figure 4(b)). Although the fragment $\mathbf{4 a}$ had a similar substructure to the hit fragment $\mathbf{1}$, such simple structure has a risk to bind in a different binding mode to that of $\mathbf{1}$. Hence, we decided to confirm whether and how the fragment bound to renin in order to carry out further chemical modification with a high degree of accuracy. The X-ray crystal structure analyses of $\mathbf{4 a}$ revealed that the monocyclic compound binds to renin in a similar manner to 1 except the interaction with the S1 site (Figure 4(b)). The pyrazine ring and the furfurylamino group bind to the $\mathrm{S} 3$ site and the $\mathrm{S} 3{ }^{\mathrm{SP}}$ site, respectively, and the $\mathrm{NH}$ of the furfurylamino group retains a hydrogen-bond with the backbone carbonyl of Gly217. Meanwhile, there is no interaction with the S1 site, which leads to a disordered flap 
structure. Since the potency of $\mathbf{4 a}$ was almost equal to that of $\mathbf{1}$ despite no interactions with the $\mathrm{S} 1, \mathrm{~S} 3$ and $\mathrm{S} 3{ }^{\mathrm{SP}}$ sites were identified as the more indispensable sites for ligand-binding compared to the S1 site. The original fragment 1 and the hit compounds in the initial library suggested that the S3 site can accommodate various kinds of aryl rings such as pyrazine and pyrimidine. The flexible positions of a nitrogen atom within an aryl ring indicate the S3 site doesn't have large electric deflection on the surface. All the hits in the initial library contained the furfurylamino group as a $\mathrm{S}^{\mathrm{SP}}$ site binder. The other substituents introduced as furan analogues, such as the methoxyethylamino and benzylamino groups, could not gain enough affinity because of the unsuited complementarity for the $\mathrm{S} 3^{\mathrm{SP}}$ site (data not shown). The furfurylamino group might fit in exactly with the $S 3^{\mathrm{SP}}$ site due to the size and polarity, because it brings a simple molecule like $\mathbf{4 a}$ in the renin binding pocket. The resulting compound $\mathbf{4 a}$, which keeps potency and holds a suitable vector for further substitution targeting the catalytic aspartate residues, represented a good lead molecule for further design and elaboration.

\section{2-3. $2^{\text {nd }}$ library for targeting the S1 site}

Having successfully transformed the core of the original hit fragment from tricyclic to the smaller and more chemically accessible monocyclic core, we revisited the 
S1 site and initiated chemical modifications to the new scaffold. The crystal structure of 4a indicated that the ortho-position of the furfurylamino group, that is the nitrogen atom (N1) at the 1-position of the pyrazine core (Figure 4(b)), was the best position for an extension towards the S1 site and further targeting the catalytic aspartate residues. The $\mathrm{N} 1$ of the pyrazine core had to be replaced by a carbon atom to introduce substituents at this position. The S3 site brings in various kinds of aryl rings according to the SAR data of the first library, and their endocyclic nitrogen atom might not be involved in essential interactions according to the crystal structures of $\mathbf{1}$ and $\mathbf{4 a}$ complexed with renin. Hence, we selected the pyridine ring as a new core due to easy availability of the reagent. The chlorine atom, which was adjacent to N1 of the pyrazine core, was omitted for the new pyridine core because it seems to sterically interfere with the binding of substituents at the original N1 position to the S1 site. Instead of the chlorine atom at the 4-position of the pyridine ring, the methyl group was incorporated in the 6-position to form a favorable contact with the S3 site. As a linker introduced to N1, we examined synthetic accessible structures such as aminocarbonyl, carboxamido, sulfonamido, aryl, anilino, and phenoxy groups. As the result of a docking study, the aminocarbonyl group was selected because its carbonyl oxygen atom was estimated to make a hydrogen bond with the hydroxy group of Thr77 and diverse chemical components can be incorporated targeting the S1 site as 
amine reagents via convenient synthesis. Those led the library synthesis of

3-2-furfurylamino-6-methylpyridine-3-carboxyamide to efficiently explore the best

building block to the $\mathrm{S} 1$ site. The second library was synthesized using the simple amide coupling with amines to 2-furfurylamino-6-methylpyridine-3-carboxylic acid $\mathbf{5}$ (scheme

2). The amines for R1 and R2 group of $\mathbf{6}$ were strategically selected by means of a docking study of a virtual library generated from about ten thousand commercially available amines. Among them, promising amines were strategically selected by docking study not only to better occupy the S1 site but also to obtain informative SAR.<smiles>Cc1ccc(C(=O)O)c(NCc2ccco2)n1</smiles>

5

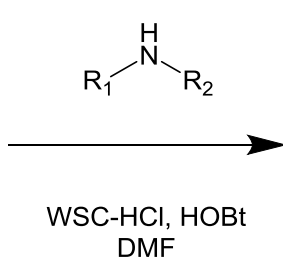

$$
\text { DMF }
$$

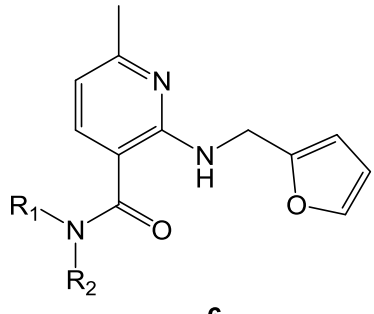

6

Scheme 2. Synthesis of the second library using amide formation of the carboxylic acids attached at the 3-position of the pyridine ring

Among the strategically selected amines, cyclic tertiary amides were in particular predicted to form favorable contacts with the S1 site. We then synthesized the derivatives as $6 \mathbf{a}$ and $\mathbf{6 b}$, which exhibited $\mathrm{IC}_{50}$ values of $11 \mu \mathrm{M}$ and $38 \mu \mathrm{M}$ respectively 
(Figure 4(c)). The crystal structure of $\mathbf{6 b}$ showed the furfurylamino group and the pyridine ring bound in an almost identical fashion to 1 (Figure 4(a, c)). The flap which was disordered before due to the lack of any specific interactions was ordered in the close conformation in an identical fashion to 1 (Figure 4(a, c)). The benzene ring of Tyr75 of the flap forms the $\mathrm{CH} / \pi$ interaction with the alkyl chain of the azepane ring similar to the endocyclic ring of the original hit fragment. In addition, the carbonyl oxygen atom of the amide of $\mathbf{6 b}$ forms a hydrogen bond with the hydroxy group of Thr77 of the flap. This polar interaction, which the original hit fragment could not make, likely helped the flap to adopt the close conformation. The close conformation of the flap substantially forms the S1 site, and the cyclic alkyl part properly occupied the hydrophobic S1 site. The SAR data of the second library showed that only a few qualified components could take appropriate complementarities of both shape and polarity. For example, the polar NH of secondary amide $\mathbf{6 d}$ caused unsuitable binding to the hydrophobic S1 site, and non-cyclic tertiary amides such as diethylamino moiety $\mathbf{6 c}$ could not bring strong enough binding to the S1 site (Supplementary Table 1). In order to bind strongly enough to renin without interactions with the catalytic residues, a perfect complementarity on both shape and polarity is strictly required. 


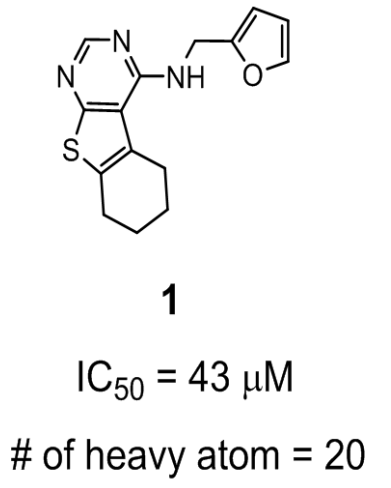

$\checkmark$

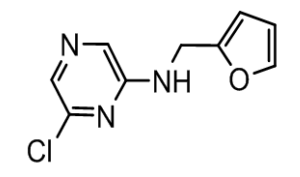

$4 a$

$65 \%$ at $100 \mu \mathrm{M}$

$\#$ of heavy atom $=14$

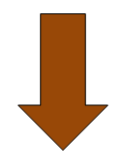

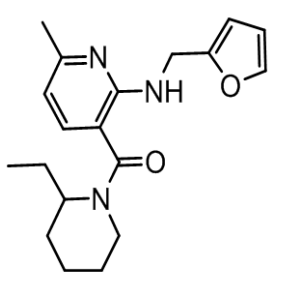

$6 a$

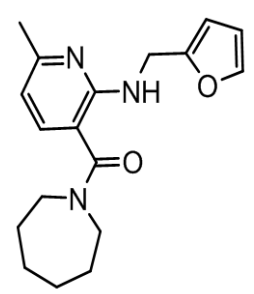

$6 b$

$\mathrm{IC}_{50}=11 \mu \mathrm{M}$

\# of heavy atom $=24$

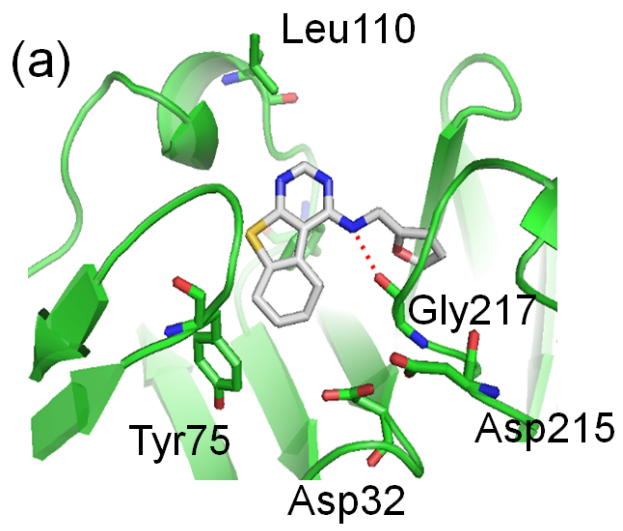

(b)

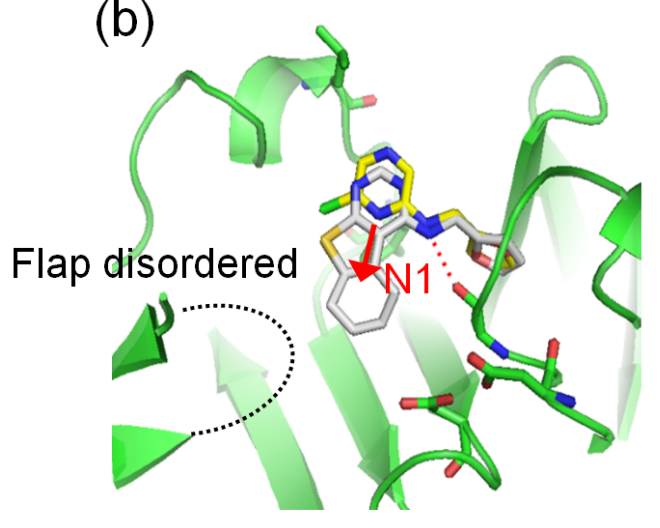

(c)

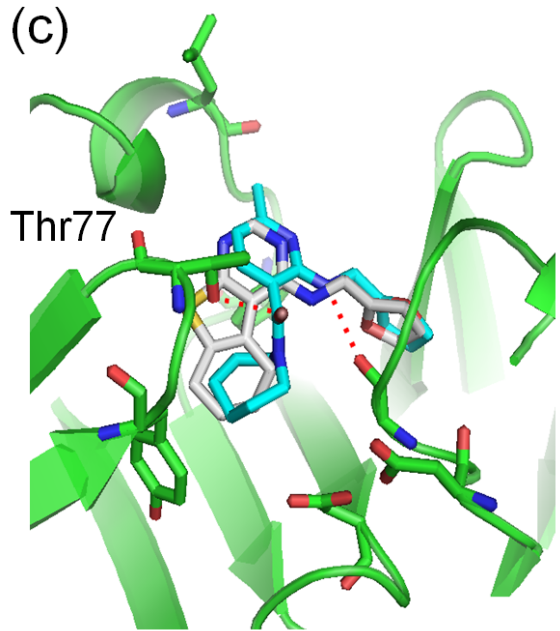

Figure 4 Progress of the fragment from hit to lead. On the left side, a schematic

representation of the inhibitors is depicted together with the IC50 values and the number of heavy atoms. On the right side, X-ray crystal structure of (a) $\mathbf{1}$ (white), (b) $\mathbf{4 a}$ (yellow), 
and (c) $\mathbf{6 b}$ (cyan) overlaid with $\mathbf{1}$ are shown. Important residues are shown as sticks.

Hydrogen-bonds are shown as red, dashed lines. [PDB entry 5SY3 for 1, 5T4S for 4a, and 5SZ9 for $\mathbf{6 b}]$

The 2-furfurylamino-6-methylpyridine-3-carboxyamide series, such as $\mathbf{6 a}$ and $\mathbf{6 b}$, derived from hit fragment $\mathbf{1}$ were considered to be promising lead compounds in terms of lead-likeness. This is not only because the series could bind to the S1, S3, and $\mathrm{S} 3{ }^{\mathrm{SP}}$ sites efficiently enough still without engaging the catalytic aspartate residues, but also because they are much more chemically accessible, which is critical to lead optimization. To better understand quality of the new lead compounds, ClogP and ligand efficiency related indices, which are important factors for lead discovery procedure, between $\mathbf{6 b}$ and $\mathbf{1}$ were compared (Table 1). The conversion of $\mathbf{1}$ to $\mathbf{6 b}$ lowered ClogP from 3.9 to 2.9 , keeping the inhibitory activity and the molecular size almost equally. Reflecting decrease of ClogP, the value of ligand-lipophilic efficiency (LLE $=\mathrm{pIC}_{50}$ Clog $\mathrm{P}^{18}$ ) moved in the direction to decrease the lipophilic component of activity, while that of ligand efficiency $\left(\mathrm{LE}=\right.$ pIC50/number of heavy atoms $\left.{ }^{19}\right)$ remained almost unchanged. In addition, the solubility and in vitro toxic profiles of $\mathbf{1}$ were improved for 6b. The higher solubility of $\mathbf{6 b}$ in JP2 was probably due to higher conformational 
flexibility of the monocyclic scaffold ${ }^{20,21}$ and CYP3A4 inhibition, hERG inhibition, and cytotoxicity were improved due to less lipophilicity (Table 2). Although the metabolic stability in human was still low, the improvement tendency was shown (Table 2). As can be speculated from the fact that $\mathbf{6 b}$ mainly binds to the hydrophobic S1-S3 sites, its lipophilicity is slightly high considering its small molecular size. It is estimated that further chemical modification with the introduction of polar parts targeting the catalytic residues might improve metabolic stability.

Table 1. Change in ligand efficiency indices of hit fragment $\mathbf{1}$ and lead $\mathbf{6 b}$

\begin{tabular}{cccccc}
\hline & $\mathrm{IC} 50(\mu \mathrm{M})$ & $\mathrm{HAC}^{\mathrm{a}}$ & $\mathrm{ClogP}^{\mathrm{b}}$ & $\mathrm{LE}^{\mathrm{c}}$ & $\mathrm{LLE}^{\mathrm{d}}$ \\
\hline $\mathbf{1}$ & 43 & 20 & 3.9 & 0.22 & 0.47 \\
$\mathbf{6 b}$ & 38 & 23 & 2.9 & 0.19 & 1.52 \\
\hline
\end{tabular}

${ }^{\mathrm{a}}$ Heavy atom count. ${ }^{\mathrm{b}}$ Calclated $\log \mathrm{P}$ by Daylight. ${ }^{\mathrm{c}}$ pIC50/HAC.

d pIC50-ClogP: the higher values of LLE means to reduce the

Table 2. The solubility, metabolic stability, and in vitro toxic profiles of $\mathbf{1}$ and $\mathbf{6 b}$

\begin{tabular}{cccccc}
\hline $\begin{array}{c}\text { Solubility in } \\
\mathrm{JP} 2^{\mathrm{a}} \\
(\mu \mathrm{g} / \mathrm{mL})\end{array}$ & $\begin{array}{c}\text { Metabolic } \\
\text { stability }^{\mathrm{b}} \text { in } \\
\text { human } \\
(\mu \mathrm{L} / \mathrm{min} / \mathrm{mg}\end{array}$ & $\begin{array}{c}\text { CYP3A4 } \\
(\% \text { inhibition } \\
\text { at } 10 \mu \mathrm{M})\end{array}$ & $\begin{array}{c}\text { hERG } \\
(\% \text { inhibition } \\
\text { at } 10 \mu \mathrm{M})\end{array}$ & $\begin{array}{c}\text { Cytotoxicity } \\
\text { ATP contents } \\
(\% \text { control } \\
\text { at } 30 \mu \mathrm{M})\end{array}$ \\
\hline $\mathbf{1}$ & 5.2 & 187 & 70.2 & 62.8 & 31.4 \\
$\mathbf{6 b}$ & 50 & 85 & 35.6 & 7.5 & 91.6 \\
\hline
\end{tabular}

${ }^{\mathrm{a}}$ Second fluid for the Disintegration test regulated in the Japanese Pharmacopoeia 15th edition (pH6.8). ${ }^{\mathrm{b}}$ In vitro metabolic clearance in human liver microsome. 


\section{Conclusions}

We successfully created the promising lead compounds $\mathbf{6 a}$ and $\mathbf{6 b}$ as renin inhibitors by using the fragment-based lead discovery technique. The highly sensitive biochemical screening of a fragment library and the advanced X-ray crystallography methods provided us the unique hit fragment 1 which had the S1 site, the S3 site, and the $\mathrm{S}^{\mathrm{SP}}$ site without any interactions with the catalytic aspartate residues. Although the fragment library has been carefully designed and contained many basic fragments, none of those were bound to the target. We concluded from this fact that those sites are essential for renin inhibition. Prior to making chemical modifications to the hit fragment, we decided to optimize the hit fragment itself with identifying additional essential site(s). This is especially important, because there was no guarantee that the hit fragment is the best starting molecule for the optimization leading to a lower-molecular-weight renin inhibitor successfully. The key elements of the best starting molecule should be high ligand efficiency and chemical accessibility. First, we identified the essential site(s) of renin by measuring the renin inhibitory activities for the substructures of the hit fragment. Second, we designed a library synthesis with simple chemistry (Scheme 1) to explore the best building blocks to occupy the essential S3 site and S3 ${ }^{\mathrm{SP}}$ site using high-throughput chemistry. We then successfully transformed the tricyclic scaffold of $\mathbf{1}$ to the simple 
monocyclic scaffold of $\mathbf{4 a}$. We deemed the fragment $\mathbf{4 a}$ as the new starting molecule for creating a novel renin inhibitor due to its simple structure and practical chemical accessibility. The X-ray crystal structure revealed that fragment $4 \mathbf{a}$ bound to the S3 site and the $\mathrm{S} 3^{\mathrm{SP}}$ site as predicted (Figure 4(b)), which helped us to design the next library synthesis. We then revisited the S1 site, and designed and synthesized 2-furfurylamino-6-methylpyridine-3-carboxyamide library (Scheme 2) resulting in the high quality lead compounds, $6 \mathbf{a}$ and $\mathbf{6 b}$. These compounds were superior to the hit fragment 1 in terms of (1) ClogP and LLE, (2) good chemical accessibility to target the S1 site and the catalytic residues, (3) good solubility and in vitro toxic profiles, and (4) preferable binding modes: In the crystal structure, $6 \mathbf{b}$ fixes the flap in the close conformation and binds tightly via a hydrogen bonding between its scaffold and the backbone carbonyl of Thr77. It should be noted here that the 2-furfurylamino-6-methylpyridine-3-carboxyamide compounds were fairly structurally novel. Even though those compounds could be synthesized by simple chemistries (Scheme 1 and 2), they were at least not commercially available. The high quality lead compounds provided the new structural insight into renin's active site and the potential to create a new generation of renin inhibitors. The successful integration of the different techniques used for fragment-based lead discovery, namely highly sensitive biochemical 
screening, advanced X-ray crystallography, high-throughput synthetic chemistry and computational chemistry led to the discovery of the promising lead compounds for the traditionally very challenging drug discovery target renin.

\section{Experimental Section}

\section{4-1. Purification of recombinant human renin}

Human preprorenin was expressed using the FreeStyle 293 Expression System (Invitrogen). The recombinant prorenin was exported by FreeStyle 293 cells into the tissue culture media. The cell culture supernatants were processed by filtration, concentration and dialysis in $20 \mathrm{mM}$ Tris- $\mathrm{HCl}$ buffer (pH8.0). Prorenin was purified using Resource Q column (GE Healthcare) and HiLoad 16/60 Superdex 200pg (GE Healthcare). Prorenin was activated to renin by trypsin digestion.

\section{4-2. Renin activity assay using microchip type capillary electrophoresis (Mobility shift assay)}

In a $384-w e l l$ plate (Nalgen-Nunc), $2 \mu$ of test compound in $100 \%$ DMSO was incubated with $28 \mu \mathrm{l}$ of enzyme in buffer ( $5 \mathrm{nM}$ renin, $20 \mathrm{mM}$ citric acid buffer ( $\mathrm{pH}$ 6.0) and $0.004 \%$ Triton-X100) at $37^{\circ} \mathrm{C}$. After $15 \mathrm{~min}, 10 \mu \mathrm{l}$ of substrate peptide 
(FITC-eAcp-Asp-Arg-Val-Tyr-Ile-His-Pro-Phe-His-Leu-Val-Ile-His-Asn-Arg-NH2, $5 \mu \mathrm{M})$ was added to each well to start the reaction. After incubation at $37^{\circ} \mathrm{C}$ for $30 \mathrm{~min}$, the reaction was terminated by addition of $40 \mu$ of stop solution ( $1 \mu \mathrm{M}$ CGP-29287, 200mM Tris-HCl (pH 8.0), 0.04\% Triton-X 100, 0.4\% Coating 3 reagent (Caliper Life Sciences)). After completion of the reaction, substrate and product peptide were separated by electrophoresis and quantified by fluorescence detection (excitation wavelength $457 \mathrm{~nm}$, measurement wavelength 530nm) using a microchip type capillary electrophoresis apparatus 250HTS (Caliper Life Sciences). The rate of substrate conversion was calculated by dividing the product peak height by the sum of the substrate and product peak height $(\mathrm{P} /(\mathrm{P}+\mathrm{S}))$. Inhibitory activity of each compound was calculated on the basis

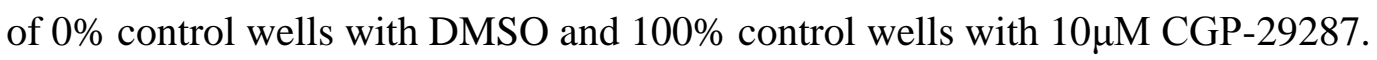

\section{4-3. Crystallography}

Apo crystals of mature human renin (aa1-340, ca. $6 \mathrm{mg} / \mathrm{mL}$ in $25 \mathrm{mM}$ Tris $\mathrm{pH}$ 7.9 and $150 \mathrm{mM} \mathrm{NaCl}$ aqueous solution) were grown by the sitting drop vapor diffusion method $^{15}$ at $20{ }^{\circ} \mathrm{C}$. Conditions for the reservoir solution were $23 \%-26 \%$ PEG600, 100 mM citric acid buffer $\mathrm{pH} 4.5$ - 6.0, or 30\% PEG600, $100 \mathrm{mM}$ citric acid buffer $\mathrm{pH} 4.5$ 6.0, $50 \mathrm{mM} \mathrm{NaH} \mathrm{PO}_{4}$. The compounds were soaked into the apo crystals at $1-10 \mathrm{mM}$, 
for 2 to 18 hours. X-ray diffraction analysis was carried out at beamline 5.0.3 of the Advanced Light Source (ALS) and at beamline ID23B of the Advanced Photon Source (APS). The data was reduced by the HKL2000 software package ${ }^{22}$. The structures were determined by the molecular replacement method using MOLREP ${ }^{23}$ of the CCP4 program suite. Multiple cycles of model building with $\mathrm{XtalView}^{24}$ or $\mathrm{COOT}^{25}$ and refinement with $\mathrm{REFMAC}^{26}$ were performed to improve model quality.

\section{4-4. Computational Study}

Docking experiments were used to predict the binding mode of the components of a virtual library and to select compounds for synthesis. Docking studies were carried out in the six step: (1) conformations of the components of the virtual library were generated using OMEGA $1.8 .1^{27}$, (2) these conformations were placed in the active site of renin, aligned to the furfurylamino group of $\mathbf{1}$ bound to renin, (3) the molecules were wobbled by rotating and/or translating them over a certain angle and distance, (4) molecules which collided with the protein surface were discarded, (5) the molecules were energy-minimized with the MMFF94s force-field using MOE 2006.08 ${ }^{28}$, and (6) their binding free energy was calculated using the MM/PBSA method. The prospective molecules were selected in terms of the calculated binding free energies and the structural 
diversity.

\section{4-5. Chemistry}

Proton nuclear magnetic resonance $\left({ }^{1} \mathrm{H}\right.$ NMR) spectra were recorded on a Brucker AV400 (400 MHz) with tetramethylsilane as internal standard. Solvents and reagents were obtained from commercial suppliers and were used without purification.

Chromatographic purification was carried out on Purif-Pack (SI $60 \mu \mathrm{M}$ or NH $60 \mu \mathrm{M}$, Fuji Silysia, LTD.) or Biotage (SI $60 \mu \mathrm{M}$, Biotage, Inc.). The yields reported were not optimized. Combined liquid chromatography-mass spectrometry LC/MS (ESI-MS) analyses were carried out using a Waters Open-Lynx system. High performance liquid chromatography analyses were performed on a Shimadzu LC-20A system. HPLC was carried out using a Gilson system employing the following conditions: Column; YMC CombiPrep Hydrosphere C-18 S-5 $\mu \mathrm{m}, 50$ x 20 mm or YMC CombiPrep Pro C-18 RS S-5 $\mu \mathrm{m}, 50$ x $20 \mathrm{~mm}$; Mobile phase: $\mathrm{MeCN} / \mathrm{H}_{2} \mathrm{O} /$ trifluoroacetic acid = 5:95:0.1 $(0 \mathrm{~min}) \rightarrow$ 5:95:0.1 $(1 \mathrm{~min}) \rightarrow$ 100:0:0.1 $(4 \mathrm{~min}) \rightarrow$ 100:0:0.1 (7.0 $\mathrm{min})$; flow rate; $20 \mathrm{ml} / \mathrm{min}$ detection; UV $220 \mathrm{~mm}$. Purity data were collected by a HPLC with Corona CAD(Charged Aerosol Detector). The column was a L-column 2 ODS (30 mm x $2.0 \mathrm{~mm}$ I.D., CERI, Japan) with a temperature of $50{ }^{\circ} \mathrm{C}$ and a flow rate of $0.5 \mathrm{~mL} / \mathrm{min}$. Mobile 
phase $\mathrm{A}$ and $\mathrm{B}$ under a neutral condition were a mixture of $5 \mathrm{mmol} / \mathrm{L}$ Ammonium acetate and $5 \mathrm{mmol} / \mathrm{L}$ ammonium acetate in $98 \%$ acetonitrile, respectively. Mobile phase A and B under an acidic condition were a mixture of $0.2 \%$ formic acid in $10 \mathrm{mmol} / \mathrm{L}$ ammonium formate and $0.2 \%$ formic acid in acetonitrile, respectively. The ratio of mobile phase B was increased linearly from $14 \%$ to $86 \%$ over $3 \mathrm{~min}, 86 \%$ over the next $1 \mathrm{~min}$.

The term "MeCN" refers to acetonitrile, "EtOAc" refers to ethyl acetate, "WSC-HCl" refers to 1-(3-dimethylaminopropyl)-3-ethylcarbodiimide hydrochloride, "HOBt" refers to 1-hydroxybenzotriazole, and " $\mathrm{CDCl}_{3}$ " refers to Chloroform- $d$.

\section{4-5-1. 6-Chloro- $N$-(2-furylmethyl)pyrazin-2-amine trifluoroacetate (4a)}

To a solution of 2,6-dichloropyrazine $(29.8 \mathrm{mg}, 0.200 \mathrm{mmol})$ in 1-methylpyrrolidin-2-one $(0.50 \mathrm{ml})$, was added 1-(2-furyl)methanamine $(97.1 \mathrm{mg}, 1.00$ mmol) and the mixture was heated at $150{ }^{\circ} \mathrm{C}$ overnight. The solvent was removed under reduced pressure, and the residue was purified by HPLC to give the title compound (37.3 mg, $0.115 \mathrm{mmol}, 58 \%)$. LC-MS: $m / z=208.0\left([\mathrm{M}-\mathrm{H}]^{-}\right)$.

\section{4-5-2. 2-[(2-Furylmethyl)amino]-6-methylnicotinic acid (5)}

A solution of 2-chloro-6-methylnicotinic acid (1.72 $\mathrm{g}, 10.0 \mathrm{mmol})$ and 
1-(2-furyl)methanamine $(1.94 \mathrm{~g}, 20.0 \mathrm{mmol})$ in 1-methylpyrrolidin-2-one $(25 \mathrm{ml})$ was heated at $150{ }^{\circ} \mathrm{C}$ overnight. The solvent was evaporated, and the residue was purified by $\mathrm{SiO}_{2}$ chromatography $(\mathrm{EtOAc} /$ hexane $=20 \%-100 \%)$ to give the title compound $(838$ $\mathrm{mg}, 3.61 \mathrm{mmol}, 36 \%) .{ }^{1} \mathrm{H} \mathrm{NMR}\left(400 \mathrm{MHz}, \mathrm{CDCl}_{3}\right) \delta 2.45(\mathrm{~s}, 3 \mathrm{H}) 4.78(\mathrm{~s}, 2 \mathrm{H}) 6.26(\mathrm{~d}$, $J=3.18 \mathrm{~Hz}, 1 \mathrm{H}) 6.32(\mathrm{dd}, J=3.18,1.71 \mathrm{~Hz}, 1 \mathrm{H}) 6.46(\mathrm{~d}, J=8.07 \mathrm{~Hz}, 1 \mathrm{H}) 7.33-7.40(\mathrm{~m}$, $1 \mathrm{H}) 8.05-8.14(\mathrm{~m}, 2 \mathrm{H})$.

\section{4-5-3.}

\section{3-[(2-Ethylpiperidin-1-yl)carbonyl]- $N$-(2-furylmethyl)-6-methylpyridin-2-amine}

(6a)

To a mixture of 2-[(2-furylmethyl)amino]-6-methylnicotinic acid (65 mg, 0.277 mmol) and 2-ethylpiperidine (48 $\mathrm{mg}, 0.420 \mathrm{mmol})$ in DMF (2.8 ml), were added HOBt (46 mg, $0.340 \mathrm{mmol})$ and WSC- $\mathrm{HCl}(65 \mathrm{mg}, 0.340 \mathrm{mmol})$ and the mixture was stirred at room temperature overnight. To the reaction mixture were added EtOAc $(10 \mathrm{ml})$ and $2 \%$ $\mathrm{NaHCO}_{3}(\mathrm{aq})(2 \mathrm{ml})$ and extraction was carried out. The water phase was washed with EtOAc $(10 \mathrm{ml})$. The combined organic phase was dried with $\mathrm{MgSO}_{4}$ and evaporated. The residue was purified by $\mathrm{SiO}_{2}$ chromatography (EtOAc/hexane $=0 \%-30 \%$ ) to give the title compound (81.9 mg, $0.250 \mathrm{mmol}, 89 \%)$. LC-MS: $m / z=328([\mathrm{M}+\mathrm{H}]+) .{ }^{1} \mathrm{H} \mathrm{NMR}$ 
$\left(400 \mathrm{MHz}, \mathrm{CDCl}_{3}\right) \delta 0.81(\mathrm{~s}, 3 \mathrm{H}) 1.38-1.62(\mathrm{~m}, 4 \mathrm{H}) 1.65-1.80(\mathrm{~m}, 4 \mathrm{H}) 2.41(\mathrm{~s}, 3 \mathrm{H})$ $2.83-2.97(\mathrm{~m}, 3 \mathrm{H}) 4.65(\mathrm{~d}, J=5.38 \mathrm{~Hz}, 2 \mathrm{H}) 5.80(\mathrm{~s}, 1 \mathrm{H}) 6.22(\mathrm{~d}, J=3.18 \mathrm{~Hz}, 1 \mathrm{H}) 6.26$ $6.34(\mathrm{~m}, 1 \mathrm{H}) 6.43(\mathrm{~d}, J=7.58 \mathrm{~Hz}, 1 \mathrm{H}) 7.19(\mathrm{~d}, J=7.58 \mathrm{~Hz}, 1 \mathrm{H}) 7.33$ (s, $1 \mathrm{H})$. Purity measurement by HPLC: $99.4 \%$.

\section{4-5-4. 3-(Azepan-1-ylcarbonyl)- $N$-(2-furylmethyl)-6-methylpyridin-2-amine (6b)}

To a mixture of 2-[(2-furylmethyl)amino]-6-methylnicotinic acid (30 mg, 0.129 mmol) and hexamethyleneimine (19 $\mathrm{mg}, 0.192 \mathrm{mmol})$ in DMF $(2.0 \mathrm{ml})$, were added HOBt (21 mg, $0.155 \mathrm{mmol})$ and WSC-HCl (30 mg, $0.156 \mathrm{mmol})$ and the mixture was stirred at room temperature overnight. To the reaction mixture were added EtOAc $(7 \mathrm{ml})$ and $2 \% \mathrm{NaHCO}_{3}$ (aq) $(1.5 \mathrm{ml})$ and extraction was carried out. The water phase was washed with EtOAc $(7 \mathrm{ml})$. The combined organic phase was dried with $\mathrm{MgSO}_{4}$ and evaporated. The residue was purified by $\mathrm{SiO}_{2}$ chromatography $(\mathrm{EtOAc} / \mathrm{hexane}=0 \%-$ $30 \%$ ) to give the title compound (30 mg, $0.0957 \mathrm{mmol}, 74 \%$ ). LC-MS: $\mathrm{m} / z=314$ $([\mathrm{M}+\mathrm{H}]+) .{ }^{1} \mathrm{H} \mathrm{NMR}\left(400 \mathrm{MHz}, \mathrm{CDCl}_{3}\right) \delta 1.55-1.86(\mathrm{~m}, 8 \mathrm{H}) 2.41(\mathrm{~s}, 3 \mathrm{H}) 3.52(\mathrm{~s}, 4 \mathrm{H})$ $4.65(\mathrm{~d}, J=5.62 \mathrm{~Hz}, 2 \mathrm{H}) 5.81(\mathrm{t}, J=5.26 \mathrm{~Hz}, 1 \mathrm{H}) 6.23(\mathrm{~d}, J=3.18 \mathrm{~Hz}, 1 \mathrm{H}) 6.27-6.34(\mathrm{~m}$, $1 \mathrm{H}) 6.43(\mathrm{~d}, J=7.58 \mathrm{~Hz}, 1 \mathrm{H}) 7.22(\mathrm{~d}, J=7.34 \mathrm{~Hz}, 1 \mathrm{H}) 7.34(\mathrm{~d}, J=1.71 \mathrm{~Hz}, 1 \mathrm{H})$. Purity measurement by HPLC: $100 \%$. 


\section{4-5-5. $N, N$-Diethyl-2-((2-furylmethyl)amino)-6-methylnicotinamide trifluoroacetate}

(6c)

To a mixture of 2-[(2-furylmethyl)amino]-6-methylnicotinic acid (13.9 mg, $0.060 \mathrm{mmol})$ and $N$-ethylethanamine $(5.3 \mathrm{mg}, 0.072 \mathrm{mmol})$ in DMF $(1.0 \mathrm{ml})$, was added a solution of HOBt $(9.7 \mathrm{mg}, 0.072 \mathrm{mmol})$ and WSC-HCl (13.8 mg, $0.072 \mathrm{mmol})$ in DMF $(0.50 \mathrm{ml})$. The mixture was stirred at room temperature overnight. To the reaction mixture, was added $2 \% \mathrm{NaHCO}_{3}(\mathrm{aq})(1.0 \mathrm{ml})$ and the crude mixture was extracted with EtOAc $(3.0 \mathrm{ml})$. The organic layer was washed with water $(2.0 \mathrm{ml})$, and evaporated. The residue was purified by HPLC to give the title compound (16.9 mg, $0.0421 \mathrm{mmol}, 70 \%$ ). LC-MS: $m / z=288.2\left([\mathrm{M}+\mathrm{H}]^{+}\right)$.

\section{4-5-6. 2-((2-Furylmethyl)amino)-6-methyl- $N$-(2-phenylpropan-2-yl)nicotinamide}

\section{trifluoroacetate $(6 \mathrm{~d})$}

To a mixture of 2-[(2-furylmethyl)amino]-6-methylnicotinic acid (13.9 mg, $0.060 \mathrm{mmol})$ and 2-phenylpropan-2-amine $(9.7 \mathrm{mg}, 0.072 \mathrm{mmol})$ in DMF (1.0 ml), was added a solution of HOBt $(9.7 \mathrm{mg}, 0.072 \mathrm{mmol})$ and WSC-HCl $(13.8 \mathrm{mg}, 0.072 \mathrm{mmol})$ in DMF $(0.50 \mathrm{ml})$. The mixture was stirred at room temperature overnight. To the reaction 
mixture, was added $2 \% \mathrm{NaHCO}_{3}(\mathrm{aq})(1.0 \mathrm{ml})$ and the crude mixture was extracted with EtOAc $(3.0 \mathrm{ml})$. The organic layer was washed with water $(2.0 \mathrm{ml})$, and evaporated. The residue was purified by HPLC to give the title compound (13.2 $\mathrm{mg}, 0.0285 \mathrm{mmol}, 48 \%$ ). LC-MS: $m / z=350.1\left([\mathrm{M}+\mathrm{H}]^{+}\right)$.

\section{4-6. ADME/Tox properties}

\section{4-6-1. Solubility}

Small volumes of the compound DMSO solutions were added to the aqueous buffer.

After incubation, precipitates were separated from by filtration through a filter plate. The filtrates were analyzed for compound in solution by HPLC analysis.

\section{4-6-2. Metabolic Stability}

Human liver microsomes were purchased from Xenotech, LLC (Lenexa, KS). An incubation mixture with a final volume of $50 \mu \mathrm{L}$ consisted of microsomes in $50 \mathrm{mmol} / \mathrm{L}$ $\mathrm{KH}_{2} \mathrm{PO}_{4}-\mathrm{K}_{2} \mathrm{HPO}_{4}$ phosphate buffer (pH 7.4) and $1 \mu \mathrm{mol} / \mathrm{L}$ test compound. The concentration of microsomal protein was $0.2 \mathrm{mg} / \mathrm{mL}$. An NADPH-generating system containing $25 \mathrm{mmol} / \mathrm{L} \mathrm{MgCl} 2,25 \mathrm{mmol} / \mathrm{L}$ glucose-6-phosphate, $2.5 \mathrm{mmol} / \mathrm{L}$ beta-NADP+ and 7.5 unit/mL glucose-6-phosphate dehydrogenase was added to the 
incubation mixture with a $20 \%$ volume of the reaction mixture to initiate the enzyme reaction. The reaction was terminated 15 and 30 minutes after the initiation of the reaction by mixing the reaction mixture with an equivalent volume of acetonitrile, followed by centrifugation at $2500 \mathrm{rpm}$ for $10 \mathrm{~min}$. The supernatant was subjected to LC/MS/MS analysis. The metabolic velocity was calculated as the slope of the concentration-time plot. The in vitro intrinsic metabolic clearance was calculated by dividing initial metabolic velocity by the test compound concentration in the incubation mixture.

\section{4-6-3. CYP inhibition}

Measurement of CYP3A4 Inhibition Activity. Incubation mixtures were prepared in a total volume of $40 \mu \mathrm{L}$ with final component concentrations as follows: $50 \mathrm{mmol} / \mathrm{L}$ phosphate buffer ( $\mathrm{pH} 7.4$ ), NADPH-generating system (5 mmol/L $\mathrm{MgCl}_{2}, 0.5 \mathrm{mmol} / \mathrm{L}$ $\beta$-NADP+, 5 mmol/L glucose-6-phosphate, and 1.5 unit/mL glucose-6-phosphate dehydrogenase), $10 \mathrm{nmol} / \mathrm{L}$ CYP3A4-expressing microsomes ( BD Biosciences), 25 $\mu \mathrm{mol} / \mathrm{L}$ testosterone, and $10 \mu \mathrm{mol} / \mathrm{L}$ test compounds. The substrates and inhibitors were dissolved in methanol and dimethylsulfoxide, respectively, and added to the incubation mixture with the final solvent concentration of $0.5 \%$. Incubations were conducted at $27^{\circ} \mathrm{C}$ for 60 minutes and terminated by adding acetonitrile. After centrifugation, aliquots of 
the supernatants were subjected to measurement of the LC/MS/MS. All incubations were made in triplicate. The activities of CYP3A4 were determined by the peak of $6 \beta$-hydroxytestosterone, respectively. The activities of test samples were expressed as the percentage of activity remaining compared with a control sample containing no inhibitor. The inhibition values were obtained as following equation:

$$
\% \text { inhibition }=100 \times(1-\text { Activity of test compound } / \text { Control activity })
$$

* Activity of test compound: activity of sample containing test

compound

Control activity: activity of control sample containing no inhibitor

\section{4-6-4. Cytotoxicity}

HepG2 cells were seeded at $2 \times 10^{\wedge} 4$ cells/well in 96-well white plate, and cultured in DMEM supplemented with $0.5 \%$ fetal bovine serum and test compound for 1 day. The cell viability was determined by the cellular ATP content. The cellular ATP content was measured by ATPLiteTM-M (PerkinElmer) according to the manufacture's instruction. ATP content was calculated to the following. ATP content $(\%$ of control $)=($ RLU of test compound / RLU of $1 \%$ DMSO) $\times 100$. 


\section{4-6-5. hERG inhibition}

hERG/CHO cells stably expressing hERG channel were purchased from Millipore (UK) Ltd. (cat. \#CYL3038). Cells were cultured at $32{ }^{\circ} \mathrm{C}, 5 \% \mathrm{CO}_{2}$ in Ham's F-12 medium supplemented with $10 \%$ fetal bovine serum, $500 \mu \mathrm{g} / \mathrm{mL}$ Geneticin (Invitrogen). The hERG inhibition assay was performed on the IonWorks Quattro (Molecular Devices) system in population patch clamp (PPC) mode. The extracellular solution was phosphate-buffered salines (PBS) with calcium and magnesium (Cat. \#14040, Invitrogen). The intracellular solution contained $140 \mathrm{mM} \mathrm{KCl,} 2 \mathrm{mM} \mathrm{MgCl} 2,1 \mathrm{mM}$ EGTA and 20 mM HEPES, pH 7.3 with $\mathrm{KOH}$. After perforation using $100 \mu \mathrm{g} / \mathrm{mL}$ amphotericin B (Sigma-Aldrich), hERG current was measured under the potential-clamp protocol (Holding potential $-80 \mathrm{mV}$, the first voltage $40 \mathrm{mV}: 2 \mathrm{sec}$, the second voltage $-50 \mathrm{mV}: 2 \mathrm{sec})$. The peaktail current before addition of the compounds was measured as the pre hERG current. Test compounds were incubated on the cells for a period of $5 \mathrm{~min}$. The peaktail current after addition of the compounds was measured as the post hERG current. \%hERG inhibition was calculated $(n=4)$ to the following. $\% \mathrm{hERG}$ inhibition $=100-($ post hERG current $/$ pre $\mathrm{hERG}$ current $) \times 100$

\section{Acknowledgement}


The authors thank Joseph Szurley, Weston Lane, Bi-Ching Sang, Kengo Okada, Noriyuki Habuka, and Aki Hirokawa for structural biology: protein preparation and X-ray data collection, and Satoshi Endo for collection of fragment library. The authors also thank the staff of the Berkeley Center for Structural Biology (BCSB), Lawrence Berkeley National Laboratory, which operates Advanced Light Source beamline 5.0.3, and the staff of GM/CA at the Argonne National Laboratory, which operates Advanced Photon Source beamline ID23-B, for their support. BCSB is supported in part by the National Institutes of Health, National Institute of General Medical Sciences, and the Howard Hughes Medical Institute. The Advanced Light Source is supported by the Director, Office of Science, Office of Basic Energy Sciences, of the U.S. Department of Energy under Contract No. DE-AC02-05CH11231.GM/CA@APS has been funded in whole or in part with Federal funds from the National Cancer Institute (ACB-12002) and the National Institute of General Medical Sciences (AGM-12006). This research used resources of the Advanced Photon Source, a U.S. Department of Energy (DOE) Office of Science User Facility operated for the DOE Office of Science by Argonne National Laboratory under Contract No. DE-AC02-06CH11357. 


\section{Accession Codes}

Atomic coordinates and structure factors have been deposited in the Protein Data Bank with codes $5 \mathrm{SY} 3$ for $\mathbf{1}, 5 \mathrm{~T} 4 \mathrm{~S}$ for $\mathbf{4 a}$, and $5 \mathrm{SZ} 9$ for $\mathbf{6 b}$, respectively.

\section{References}

1. Ress, D. C.; Congreve, M.; Murray, C. W.; Carr, R. Nat. Rev. Drug Discov. 2004, 3, $660-672$.

2. MacGregor, G. A.; Markandu, N. D.; Roulston, J. E.; Jones, J. C.; Morton, J. J. Nature 1981, 291, 329.

3. Fisher, N. D.; Hollenberg, N. K. J. Am. Soc. Nephrol. 2005, 16, 592.

4. Webb, R. L.; Schiering, N.; Sedrani, R,; Maibaum, J. J. Med. Chem. 2010, 53, 7490

5. De Gasparo, M.; Cumin, F.; Nussberger, J.; Guyenne, T. T.; Wood, J. M.; Menard, J. Br. J. Clin.

Pharmacol. 1989, 27, 587.

6. Kleinbloesem, C. H.; Weber, C.; Fahrner, E.; Dellenbach, M.; Welker, H.; Schroter, V.; Belz, G. G. Clin. Pharmacol. Ther. 1993, 53, 585.

7. Oh, B-H.; Mitchell, J.; Herron, J. R.; Chung, J.; Khan, M.; Keefe, D. L. J. Am. Coll. Cardiol. 2007, 49, 1157-1163. 
8. Cohen, N-C. Chem. Biol. Drug. Des. 2007, 70, 557-565.

9. Maibaum, J.; Stutz, S.; Göschke, R.; Rigollier, P.; Yamaguchi, Y.; Cumin, F.;

Rahuel, J.; Baum, H.-P.; Cohen, N.-C.; Schnell, C. R.; Fuhrer, W.; Gruetter, M. G.;

Schilling, W.; Wood, J. M. J. Med. Chem. 2007, 50, 4832-4844.

10. Göschke, R.; Stutz, S.; Rasetti, V.; Cohen, N-C.; Rahuel, J.; Rigollier, P.; Baum, H-P.; Forgiarini, P.; Schnell, C. R.; Wagner, T.; Gruetter, M. G.; Fuhrer, W.; Schilling, W.; Cumin, F.; Wood, J. M.; Maibaum, J. J. Med. Chem. 2007, 50, 4818-4831.

11. Tong, L.; Pav, S.; Lamare, D.; Pilote, L.; LaPlante, S.; Anderson, P. C.; Jung, G. J. Mol. Biol. 1995, 250, 211.

12. Viera, E.; Binggeli, A.; Breu, V.; Bur, D.; Fischli, W.; Güller, R.; Hirth, G.; Märki, H. P.; Müller, M.; Oefner, C.; Scalone, M.; Stadler, H.; Wilhelm, M.; Wostl, W. Bioorg. Med. Chem. Lett. 1999, 9, 1397-1402.

13. Sarver, R. W.; Peevers, J.; Cody, W. L.; Ciske, F. L.; Dyer, J.; Emerson, S. D.; Hagadorn, J. C.; Holsworth, D. D.; Jalaie, M. Anal. Biochem. 2007, 360, 30-40.

14. Ostermann, N.; Ruedisser, S.; Ehrhardt, C.; Breitenstein, W.; Marzinzik, A.; Jacoby, E.; Vangrevelinghe, E.; Ottl, J,; Hartwieg, J. C. D.; Cumin, F.; Haissiepen, U.; Trappe, T.; Sedrani, R.; Geisse, S.; Gerhartz, B.; Richert, P.; Francotte, E.; Wagner, T.; Krömer, M.; Kosaka, T.; Webb, R. L.; Rigel, D. F.; Maibaum, J.; Baeschlin, D. J. J. 
Med. Chem. 2013, 56, 2196-2206.

15. Dhanaraj, V.; Dealwis, C. G.; Frazao, C.; Badasso, M.; Sibanda, B. L.; Tickele, I. J.;

Cooper, J. B.; Driessen, H. P. C.; Newman, M.; Aguilar, C.; Wood, S. P.; Blundell, T. L.;

Hobart, P. M.; Geoghegan, K. F.; Ammirati, K. J.; Danley, D. E.; O'Connor, B. A.;

Hoover, D. J. Nature 1992, 357, 466.

16. Wang, P. G. High-Throughput Analysis in the Pharmaceutical Industry; CRC Press:

Oxford, U. K. 2008.

17. Fogolari, F.; Brigo, A.; Molinari, H. Biophys. J. 2003, 85, 159.

18. Leeson, P.; Springthorpe, B. Nat. Rev. Drug Discov. 2007, 6, 881.

19. Hopkins, A. L.; Groom, C. R.; Alex, A. Drug Discov. Today 2004, 9, 430.

20. Ishikawa, M.; Hashimoto, Y. J. Med. Chem. 2011, 54, 1539.

21. Delaney, J. S. J. Med. Inf. Comput. Sci. 2004, 44, 1000.

22. Otwinowski Z.; Minor W. Methods Enzymol. 1997, 276, 307.

23. Vagin, A.; Teplyakov A. J. Appl. Cryst. 1997, 30, 1022.

24. McRee D. E. J. Struct. Biol. 1999, 125, 156.

25. Emsley, P.; Cowtan, K. Acta. Cryst. D 2004, 60, 2126.

26. Winn M. D.; Murshudov G. N.; Papiz M. Z. Methods Enzymol. 2003, 374, 300.

27. Openeye Scientific Software, Santa Fe, USA (http://www.eyesopen.com). 
28. Chemical Computing Group Inc., Canada (http://www.chemcomp.com). 
S3

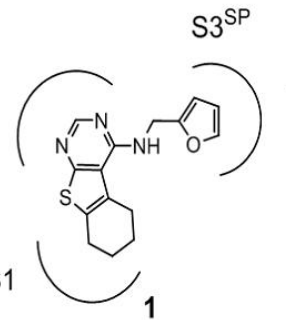

X-ray crystallography

rh-renin

$\mathrm{IC}_{50}=43 \mu \mathrm{M}$

human metabolic stability: low

CYP3A4: strong inhibition

hERG: strong inhibition

Cytotoxity: high

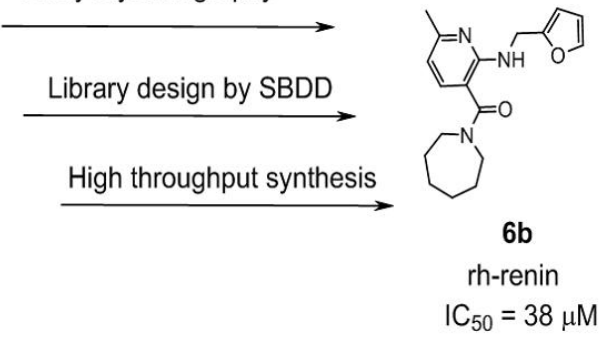

human metabolic stability: middle

CYP3A4: weak inhibition

hERG: weak inhinition

Cytotoxity: weak

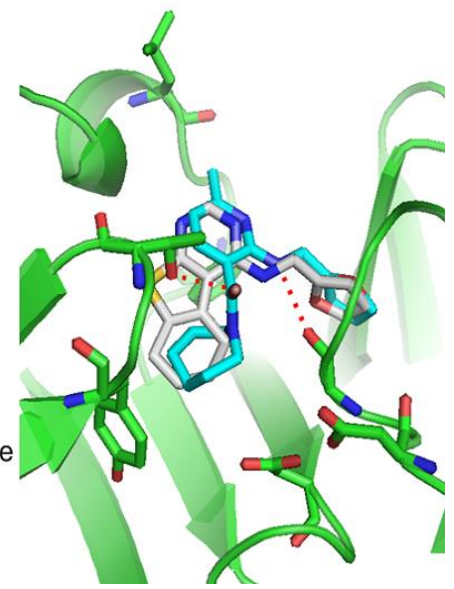

